\title{
An Axiomatic Semantics for the Synchronous Language Gentzen *
}

\author{
Simone Tini \\ Dipartimento di Informatica, Università di Pisa, Corso Italia 40, 56125, Pisa, Italy
}

\begin{abstract}
We propose an axiomatic semantics for the synchronous language Gentzen, which is an instantiation of the paradigm Timed Concurrent Constraint Programming. We view Gentzen as a prototype of the class of state-oriented synchronous languages, since it offers the basic constructs that are shared by the languages in the class. Since synchronous concurrency cannot be simulated by arbitrary interleaving, we cannot exploit "head normal forms", on which axiomatic theories for asynchronous process calculi are based.
\end{abstract}

\section{Introduction}

Synchronous languages 4,11 have been proposed for programming reactive systems 14, i.e. systems which maintain an ongoing interaction with their environment at a rate controlled by it. These languages are based on the synchronous hypothesis [8], which states that a reactive system is able to react instantaneously and in no time to stimuli from the external environment, so that outputs from the system are available as soon as inputs from the environment are. The synchronous hypothesis is indeed an abstraction and amounts to requiring that the system is able to react to an input before the subsequent input arrives.

One of the main features of the synchronous hypothesis is that "it reconciles concurrency with determinism" [8], in the sense that system components running in parallel are perfectly synchronized and cannot arbitrarily interleave, because each of them reacts whenever an input from the environment arrives.

In $\left[\begin{array}{llllll}8 & 12 & 16 & 21,3\end{array}\right]$ several state-oriented synchronous languages have been proposed for programming reactive systems where control handling aspects are predominant. These languages have been endowed with operational semantics [3. 7131622 and with denotational semantics 102022 23. We believe that it is worth developing also axiomatic semantics for such languages, in order to characterize behavioral equivalent programs. Possible applications of such semantics are, among others, transformation of programs and proof by rewriting.

Axiomatic theories are well established in the field of asynchronous process description languages since the eighties [6.17], and, for languages in suitable classes, algorithms have been developed to obtain axiomatizations in a syntaxdriven way (as examples, see [12]). A central idea in the mentioned papers is

\footnotetext{
* Research partially supported by ESPRIT Working Group COTIC, Project Number 23677, and by MURST Progetto Cofinanziato TOSCA.
} 
that concurrency can be simulated by interleaving: concurrent processes can be reduced to head normal forms, i.e. nondeterministic choices of sequential processes.

Since in the synchronous setting processes running in parallel are synchronized and run at the same rate, concurrency cannot be simulated by interleaving and head normal forms for synchronous languages have no meaning. So, developing axiomatic theories for synchronous languages cannot be done by trivially adapting what has been already done in the field of asynchronous languages.

In this paper we develop an axiomatic theory for the language Gentzen, which is an instantiation of the paradigm Timed Concurrent Constraint Programming [22]. We view Gentzen as a prototype of the class of state-oriented synchronous languages, because it offers the basic constructs that are shared by all languages in this class and that are not offered by asynchronous languages. Axiomatic theories for any other state-oriented synchronous language can be obtained by extending our work to deal with constructs that characterize such a language.

\subsection{Gentzen: A Prototype of State-Oriented Synchronous Languages}

A Gentzen program maintains an ongoing interaction with its environment by communicating through binary signals. No distinction between input and output signals is done: a set of signals $\Pi$ is assumed and signals in $\Pi$ can be sensed and broadcast both by the program and by the environment. Gentzen assumes a discrete notion of time, i.e. time is a sequence of instants. At every instant the external environment stimulates the program by broadcasting a set of signals; the program reacts immediately and broadcasts a set of signals to the environment.

We assume the syntax for Gentzen given in [21]:

$$
A::=\operatorname{skip}|\operatorname{tell} a| \text { if } a \text { then } A \mid \text { if } \bar{a} \text { then } A|\operatorname{next} A| A \| A|\operatorname{rec} P . A| P
$$

where $a, A$ and $P$ range over binary signals, agents and recursion variables, respectively. Notice that we do not use the same notation of [21. We use rec instead of " $\mu$ ", and we write if $\bar{a}$ then $A$ for if $a$ else $A$.

We will denote by $\equiv$ the syntactic identity over agents.

Agent skip does nothing at each instant: it reacts to every input by responding with the empty output. Agent tell $a$ broadcasts the signal $a$ and then terminates, i.e. it behaves as skip. Agent if $a$ then $A$ behaves as $A$ if the environment broadcasts $a$, whereas if $\bar{a}$ then $A$ behaves as $A$ if the environment does not broadcast $a$. Note that if $a$ is present (resp. absent) then if $a$ then $A$ (resp. if $\bar{a}$ then $A$ ) starts $A$ immediately. Agent next $A$ will start $A$ at the next instant. Agent $A_{1} \| A_{2}$ is the synchronous parallel composition of $A_{1}$ and $A_{2}$. Signals broadcast by $A_{1}$ (resp. $A_{2}$ ) can be detected immediately by $A_{2}$ (resp. $A_{1}$ ). Construct rec is the recursion construct. To be sure that at each instant the computation is finite, it is required that recursion be guarded, i.e. that recursion variables appear in bodies of next (see [22] for further discussion). 
According to the synchronous hypothesis, Gentzen constructs take no time, except next which takes precisely one unit of time. So, Gentzen offers the constructs that are shared by all synchronous languages: a mechanism to detect the instantaneous presence/absence of signals (if _ then _), a mechanism to broadcast instantaneously signals (tell__), a mechanism to delay the computation for one instant (next _), synchronous parallel composition (with instantaneous communication between parallel components) and guarded recursion.

Despite its simplicity, Gentzen is sufficiently expressive to embed compositionally the state-oriented synchronous language Argos [16] and the dataflow synchronous language Lustre [9] (see [25] for the proof).

\subsection{Technical Development of the Paper}

Technically, we give a structural operational semantics [19] for Gentzen in terms of a labeled transition system (LTS), with LTS states corresponding to agents and LTS transitions corresponding to agent reactions, and then we consider the bisimulation on the LTS as a behavioral equivalence over Gentzen agents. Bisimulation on our LTS is a congruence and our LTS reflects the input/output behavior of agents (in the sense that the LTS reflects the operational semantics of Gentzen given in [22]). So, bisimilar agents are distinguished neither by any Gentzen context nor by the external environment. Therefore, we believe that bisimulation is a reasonable notion of behavioral equivalence.

To axiomatize Gentzen, we provide a system of axioms defining an equality relation over agents. We prove that this equality relation is sound and complete modulo bisimulation, in the sense that two arbitrary agents are equated if and only if they are bisimilar. Our axiom system consists of a finite set of unconditional axioms plus the recursive specification principle [517]. (Since concurrency cannot be simulated by interleaving, to have a finite axiomatization we do not need the auxiliary operator "left merge" [6], which, as it has been proved in [18], is needed to have finite axiomatizations of asynchronous process algebras.)

To prove the completeness of our axiomatization, we introduce a notion of normal form of agents, we prove that any agent can be transformed into a bisimilar normal form by applying our axioms, and we prove that bisimilar normal forms are equated by our axioms. The idea of exploiting normal forms to prove completeness of axiomatizations is well established since the work of Milner [17]. But, contrarily to those of Milner, our normal forms contain the construct of parallel composition. Our normal forms are of the form $A_{1}\|\ldots\| A_{n}$, where $A_{1}, \ldots, A_{n}$ are sequential agents ("\|" is commutative and associative). The syntactic structure of our normal forms is such that, given a normal form $A_{1}\|\ldots\| A_{n}$ and an arbitrary input from the environment, $A_{1}, \ldots, A_{n}$ react, but at most one of them will be able to react to the next input.

\section{The Labeled Transition System}

In this section we propose an LTS as an operational semantic model for Gentzen. LTS states correspond to agents, LTS transitions correspond to agent reactions, 
and LTS labels carry information on causality among signals, i.e. labels highlight causes of signals broadcast by agents. These causes consist in the presence/absence of other signals.

We will prove that our LTS permits to recover the input/output behavior of agents (see Prop. 1, Sect. 2.2) and to detect whether instantaneous communications give rise to nonreactivity, i.e. the inability of an agent to react to a given input, and nondeterminism (see Prop. 2] Sect. 2.2).

Example 1. The agent $A \equiv$ if $\bar{a}$ then tell $a$ is non-reactive if the environment does not supply a. In fact, $A$ broadcasts a iff a is absent at the same instant, so no reaction is admissible. The agent $B \equiv$ if $\bar{a}$ then tell $b \|$ if $\bar{b}$ then tell $a$ is nondeterministic if the environment supplies neither a nor $b$. In fact, either the left branch of $B$ broadcasts $b$ and the right branch does not broadcast $a$, or the right branch of $B$ broadcasts $a$ and the left branch does not broadcast $b$.

Both reactivity and determinism are decidable (see [22] or Prop. 2, Sect. 2.2). Only reactive and deterministic agents are accepted in 22 . (Note that [22] requires only reactivity and nondeterminism; it does not require the more restrictive property of constructiveness recently defined in [7].) Reactivity and determinism are required also in all state-oriented synchronous languages except the specification language Statecharts [12, which admits nondeterminism.

\subsection{The Definition of the LTS}

We let $a, b$ range over the set of signals $\Pi$ and $\pi$ range over subsets of $\Pi$. We denote with $\bar{a}$ the absence of $a$ and, with abuse of notation, we define $\overline{\bar{a}}=a$. We denote with $\bar{\Pi}$ the set $\{\bar{a} \mid a \in \Pi\}$, and we let $\gamma$ range over $\Pi \cup \bar{\Pi}$.

An event $E$ (over $\Pi$ ) is a subset of $\Pi \cup \bar{\Pi}$ such that there is no signal $a$ such that both $a \in E$ and $\bar{a} \in E$. It is interpreted as the assumption that every signal $a$ with $a \in E$ (resp. $\bar{a} \in E)$ is present (resp. absent).

Definition 1. Given an event $E$ and a symbol $u \in \Pi \cup\{n\}$, the pair $(E, u)$ is a causality term with $E$ as cause and $u$ as action.

Let us explain Def. 1 Given a signal $a \in \Pi$, the action $a$ refers to the act (by some agent tell $a$ ) of broadcasting the signal $a$. The action $n$ refers to the act (by some agent next) of pausing for one instant of time. Now, given a causality term $(E, u)$, the action $u \in \Pi \cup\{n\}$ is performed by an agent that is embedded in the body of some agent if $b$ then _ (resp. if $\bar{b}$ then _) for every $b \in E$ (resp. $\bar{b} \in E)$. So, if $u=a$ then $(E, u)$ reflects causality among signals, i.e. it reflects that $a$ is broadcast because each $b \in E$ (resp. $\bar{b} \in E)$ is present (resp. absent).

Definition 2. $A$ (LTS) label is a pair $l=\left\langle\mathcal{E}_{l}, \mathcal{N}_{l}\right\rangle$ such that:

- $\mathcal{E}_{l}$ is a set of causality terms s.t. $\bigcup_{(E, u) \in \mathcal{E}_{l}} E \cup \bigcup_{(E, a) \in \mathcal{E}_{l}, a \in \Pi}\{a\}$ is an event;

$-\mathcal{N}_{l}$ is a set of causality terms s.t. for no $\left(E^{\prime}, u\right) \in \mathcal{N}_{l}$ we have that $E^{\prime} \subseteq$ $\bigcup_{(E, u) \in \mathcal{E}_{l}} E \cup \bigcup_{(E, a) \in \mathcal{E}_{l}, a \in \Pi}\{a\}$. 
The set of labels is denoted by $\mathcal{L}$. A transition $A \stackrel{l}{\longrightarrow} A^{\prime}$ corresponds to the reaction of the agent $A$ which performs the actions represented by the causality terms in $\mathcal{E}_{l}$ and does not perform the actions represented by the causality terms in $\mathcal{N}_{l}$. Causality terms in $\mathcal{E}_{l}$ are needed to recover the input/output behavior of $A$ and to discover whether $A$ is reactive and deterministic. The rôle of $\mathcal{N}_{l}$ will be explained in the following.

Table 1. The transition system specification for Gentzen.

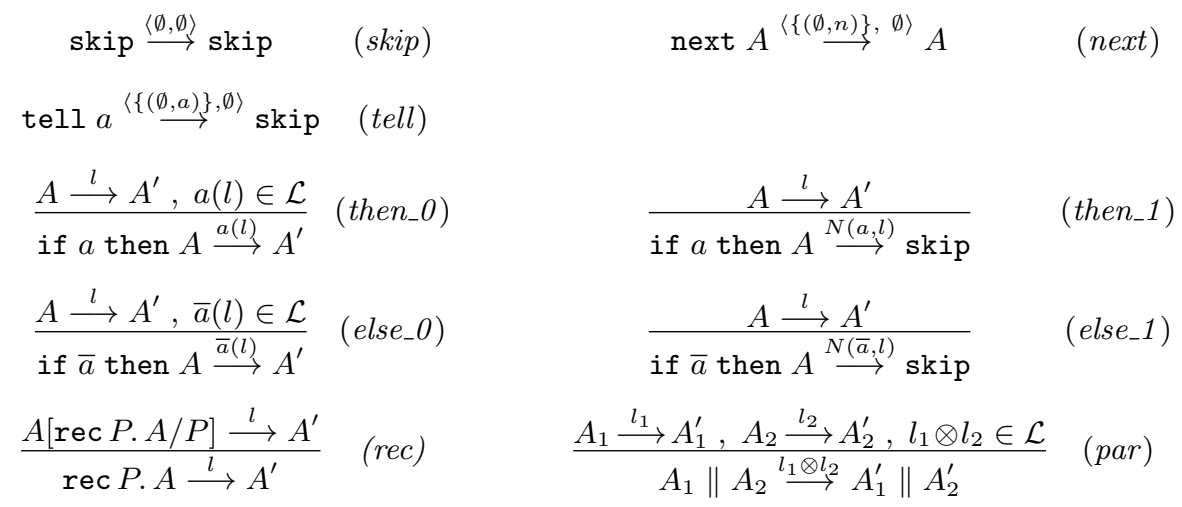

The LTS is defined by the transition system specification in Table 1.

Rule skip states that skip performs no action at every instant.

Rule next states that next $A$ performs the pausing action $n$ and will behave as $A$ at the next instant.

Rule tell states that tell $a$ broadcasts $a$ and will behave as skip. The causality term $(\emptyset, a)$ expresses that the action of broadcasting $a$ is performed independently of the presence/absence of signals in the environment.

Before explaining rules for if _then _, we introduce some more notations.

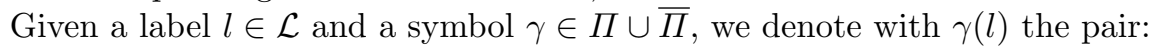

$$
\gamma(l)=\left\langle\left\{(E \cup\{\gamma\}, b) \mid(E, b) \in \mathcal{E}_{l}\right\},\left\{(E \cup\{\gamma\}, b) \mid(E, b) \in \mathcal{N}_{l}\right\}\right\rangle
$$

and with $N(\gamma, l)$ the pair:

$$
N(\gamma, l)=\left\langle\emptyset,\left\{(E \cup\{\gamma\}, b) \mid(E, b) \in \mathcal{E}_{l} \cup \mathcal{N}_{l}\right\}\right\rangle .
$$

Rule then_ 0 states that if $a$ is present then if $a$ then $A$ reacts as $A$. The label $a(l)$ highlights that actions performed by agents in the body of $A$ require the presence of $a$. Rule then_ 1 states that if $a$ is absent then if $a$ then $A$ terminates.

Example 2. Given $A \equiv$ if $a$ then tell $b$, rule then_O implies $A \stackrel{l_{0}}{\longrightarrow}$ skip, $l_{0}=\langle\{(\{a\}, b)\}, \emptyset\rangle$, and rule then_1 implies $A \stackrel{l_{1}}{\longrightarrow}$ skip, $l_{1}=\langle\emptyset,\{(\{a\}, b)\}\rangle$. 
Now, we are able to explain the rôle of the second component of labels. Let us consider the label $l_{1}$ of Example 2 The causality term $(\{a\}, b)$ in $\mathcal{N}_{l_{1}}$ keeps track of the fact that the reaction represented by the transition labeled by $l_{1}$ is performed because $a$ is absent. In principle, one could keep track of this information in other ways. As an example, it could seem to be reasonable to remove $\mathcal{N}_{l_{1}}$ and to add the causality term $(\{\bar{a}\}, s)$ to $\mathcal{E}_{l_{1}}$, with $s$ denoting some idle action. But, with a similar choice, agents if $a$ then if $b$ then $A$ and if $b$ then if $a$ then $A$ would not be bisimilar, despite they are intuitively equivalent. On the contrary, our choice guarantees that these agents are bisimilar.

Rules else_O $_{-}$and else_ 1 are analogous to then_O and then_1, respectively.

Rule par states that $A_{1} \| A_{2}$ performs both reactions of $A_{1}$ and $A_{2}$. The partial function $\otimes: \mathcal{L} \times \mathcal{L} \rightarrow \mathcal{L}$ is defined as follows:

$$
l_{1} \otimes l_{2}=\left\langle\mathcal{E}_{l_{1}} \cup \mathcal{E}_{l_{2}}, \mathcal{N}_{l_{1}} \cup \mathcal{N}_{l_{2}}\right\rangle .
$$

Finally, rule rec is a standard recursion rule.

\subsection{Correspondence with the Operational Semantics of 22}

We say that a set of signals $\pi \subseteq \Pi$ triggers a transition $A \stackrel{l}{\longrightarrow} A^{\prime}$ if the transition represents the reaction of $A$ to an environment prompting $\pi$. Formally, let us assume a label $l$ such that the causality terms in $\mathcal{E}_{l}$ having actions in $\Pi$ are $\left(E_{1}, a_{1}\right), \ldots,\left(E_{n}, a_{n}\right)$. We say that $\pi$ triggers $A \stackrel{l}{\longrightarrow} A^{\prime}$ iff there exists a sequence of sets of signals $\pi_{1} \subseteq \ldots \subseteq \pi_{n+1}$ such that:

1. $\pi_{1}=\pi$;

2. $\pi_{i+1}=\pi_{i} \cup\left\{a_{i}\right\}, 1 \leq i \leq n$;

3. $\left(E_{i} \cap \Pi\right) \subseteq \pi_{i}$ and $\overline{E_{i} \cap \bar{\Pi}} \cap \pi_{n+1}=\emptyset, 1 \leq i \leq n$;

4. both $E \cap \Pi \subseteq \pi_{n+1}$ and $\overline{E \cap \bar{\Pi}} \cap \pi_{n+1}=\emptyset$, for every $(E, u) \in \mathcal{E}_{l}$;

5. either $E \cap \Pi \nsubseteq \pi_{n+1}$ or $\overline{E \cap \bar{\Pi}} \cap \pi_{n+1} \neq \emptyset$, for every $(E, u) \in \mathcal{N}_{l}$.

Intuitively, $\pi$ triggers $A \stackrel{l}{\longrightarrow} A^{\prime}$ if $\pi$ enables a chain of agents tell $a_{i}, 1 \leq i \leq n$, so that signals in $\pi_{n+1}=\pi \cup \bigcup_{1<i<n}\left\{a_{i}\right\}$ are in the environment (cond. 13 ), all pausing actions represented by causality terms $(E, n)$ in $\mathcal{E}_{l}$ are enabled by $\pi_{n+1}$ (cond. 4), and no action represented by a causality term $(E, u)$ (with $u \in\{n\} \cup \Pi)$ in $\mathcal{N}_{l}$ is enabled by $\pi_{n+1}$ (cond. 5).

The following propositions have been proved in [26].

Proposition 1. A set of signals $\pi$ triggers a transition $A \stackrel{l}{\longrightarrow} A^{\prime}$ if and only if the agent $A$ reacts to the set of signals $\pi$ so that it will behave as $A^{\prime}$ at the next instant and the resulting environment contains signals $\pi \cup \bigcup_{(E, a) \in \mathcal{E}_{l}, a \in \Pi}\{a\}$.

Proposition 2. An agent $A$ is reactive and deterministic if and only if, for any agent $B$ such that $A \stackrel{l_{1}}{\longrightarrow} \ldots \stackrel{l_{n}}{\longrightarrow} B, n \geq 0$, every set of signals $\pi$ triggers precisely one transition $B \stackrel{l}{\longrightarrow} B^{\prime}$. 
Example 3. Given the agent $A$ of Example 1, we have that $A \stackrel{l}{\longrightarrow}$ skip, with $l=\langle\emptyset,\{(\{\bar{a}\}, a)\}\rangle$, is the unique transition from $A$. (Note that $A \stackrel{l^{\prime}}{\rightarrow}$ for $l^{\prime}=$ $\langle\{(\{\bar{a}\}, a)\}, \emptyset\rangle$, since $l^{\prime}$ violates the first condition of Def. Q and is not in $\mathcal{L}$.) $N o$ set $\pi \subseteq \Pi$ with $a \notin \pi$ triggers the transition. It follows that $A$ is non-reactive. Given the agent $B$ of Example 1, we have that $B \stackrel{l_{1}}{\longrightarrow}$ skip and $B \stackrel{l_{2}}{\longrightarrow}$ skip, with $l_{1}=\langle\{(\{\bar{a}\}, b)\},\{(\{\bar{b}\}, a)\}\rangle$ and $l_{2}=\langle\{(\{\bar{b}\}, a)\},\{(\{\bar{a}\}, b)\}\rangle$. The empty set of signals triggers both transitions. It follows that $B$ is nondeterministic.

Note that the part of LTS reachable from a given state $A$ is finite. This fact and Prop. 2 imply that reactivity and determinism of agents are decidable.

\subsection{Removing Redundant Information from Labels}

Although the LTS we have defined serves our purpose in reflecting the operational semantics of agents given in [22, we are not satisfied with it. In fact, it discriminates agents that are intuitively equivalent. As an example, agents $A$ and $A \|$ if $a$ then $A$ are not bisimilar. Our aim is now to remedy this situation.

Let del $: \mathcal{L} \rightarrow \mathcal{L}$ be the function removing redundant information from labels such that:

- del removes from $\mathcal{E}_{l} \cup \mathcal{N}_{l}$ each $(E, u)$ s.t. $\left(E^{\prime}, u\right) \in \mathcal{E}_{l} \cup \mathcal{N}_{l}$ and $E \supset E^{\prime}$;

- del removes from $\mathcal{E}_{l} \cup \mathcal{N}_{l}$ every pair of causality terms $(E \cup\{a\}, n)$ and $(E \cup\{\bar{a}\}, n)$ and adds the causality term $(E, n)$ either to $\mathcal{E}_{l}$, if $(E \cup\{a\}, n)$ or $(E \cup\{\bar{a}\}, n)$ were in $\mathcal{E}_{l}$, or to $\mathcal{N}_{l}$, if both $(E \cup\{a\}, n)$ and $(E \cup\{\bar{a}\}, n)$ were in $\mathcal{N}_{l}$.

- del replaces each causality term $(E \cup\{\gamma\}, n) \in \mathcal{E}_{l} \cup \mathcal{N}_{l}$ by $(E, n)$, provided that $\left(E^{\prime} \cup\{\bar{\gamma}\}, n\right) \in \mathcal{E}_{l} \cup \mathcal{N}_{l}$ and $E^{\prime} \subset E$.

If both $(E, u)$ and $\left(E^{\prime}, u\right)$ appear in $l$ and $E \supset E^{\prime}$, then the former causality term carries redundant information. In fact, it is sufficient that the environment prompts signals as assumed by $E^{\prime}$ to have the action represented by $u$. Intuitively, this corresponds to have that $A$ is equivalent to $A \|$ if $\gamma$ then $A$.

If both $(E \cup\{a\}, n)$ and $(E \cup\{\bar{a}\}, n)$ appear in $l$, then they are replaced by $(E, n)$ because it is sufficient that the environment prompts signals as assumed by $E$ to have the pausing action represented by $n$. Intuitively, this corresponds to have that next $A$ is equivalent to if $a$ then next $A \|$ if $\bar{a}$ then next $A$.

Note that $A \equiv$ if $a$ then tell $b \|$ if $\bar{a}$ then tell $b$ is not equivalent to $A^{\prime} \equiv$ tell $b$. In fact, agent $B \equiv$ if $b$ then tell $a$ discriminates them, since $A^{\prime} \| B$ is deterministic and reactive, whereas $A \| B$ is not. For this reason, given $b \in \Pi$, del does not replace $(E \cup\{a\}, b)$ and $(E \cup\{\bar{a}\}, b)$ by $(E, b)$.

If both $(E \cup\{\gamma\}, n)$ and $\left(E^{\prime} \cup\{\bar{\gamma}\}, n\right)$ appear in $l$ and $E^{\prime} \subset E$, then the former causality term is replaced by $(E, n)$, because it is sufficient that the environment prompts signals as assumed by $E$ to have the action represented by $n$.

Now, we will consider the LTS obtained by applying function del to every label. This corresponds to replace $a(l)$ by $\operatorname{del}(a(l)), \bar{a}(l)$ by $\operatorname{del}(\bar{a}(l)), N(a, l)$ by $\operatorname{del}(N(a, l)), N(\bar{a}, l)$ by $\operatorname{del}(N(\bar{a}, l))$ and $l_{1} \otimes l_{2}$ by $\operatorname{del}\left(l_{1} \otimes l_{2}\right)$ in Table 1

It is immediate that Prop. [1] and Prop. 2 are still valid. 


\subsection{Bisimulation}

In order to relate agents having the same input/output behavior, we consider the bisimulation on the states of the LTS. Given agents $A_{1}$ and $A_{2}$ such that $A_{1} \approx A_{2}$, the external environment is not able to distinguish between them. In fact, at each instant, if $A_{1}$ and $A_{2}$ are stimulated with the same set of signals, then they respond by broadcasting the same signals. Moreover, $A_{1}$ is reactive and deterministic if and only if $A_{2}$ is. (These facts are consequences of Prop. 1 and Prop. 2.) We show now that no Gentzen context is able to discriminate them, i.e. Gentzen constructs preserve bisimulation.

Theorem 1. The bisimulation on Gentzen agents is a congruence.

Proof. Table 1 contains a recursion rule and only rules in the well known de Simone format [24. So, Gentzen is a de Simone language and, as it has been proved in [24], this guarantees that bisimulation is a congruence.

\subsection{Some Remarks}

The structure of our LTS labels could seem too heavy. One may wonder whether LTS labels consisting of pairs 〈"received signals", "produced signals"〉 would be sufficient. Unfortunately, such a simple solution must be rejected, since labels do not carry information on signal causality, which is needed to define a semantics of a synchronous language in a compositional way (see [15] for a detailed discussion).

Example 4. Assume agents $A \equiv$ if $a$ then tell $b \|$ if $c$ then tell $d$ and $A^{\prime} \equiv$ if $a$ then if $c$ then $(\operatorname{tell} b \|$ tell $d$ ). Let $A \stackrel{l}{\longrightarrow}$ skip $\|$ skip and $A^{\prime} \stackrel{l}{\longrightarrow}$ skip $\|$ skip, with $l=\langle\{a, c\},\{b, d\}\rangle$, be the transitions representing the reactions of $A$ and $A^{\prime}$ to input $\{a, c\}$, respectively. Let $B \equiv$ if $b$ then tell $c$ and $B \stackrel{l^{\prime}}{\longrightarrow}$ skip, with $l^{\prime}=\langle\{b\},\{c\}\rangle$, be the transition representing the reaction of $B$ to input $\{b\}$. The transition $A \| B \stackrel{l^{\prime \prime}}{\longrightarrow}$ (skip $\|$ skip) $\|$ skip, with $l^{\prime \prime}=\langle\{a\},\{b, c, d\}\rangle$, must be obtained by combining $A \stackrel{l}{\longrightarrow}$ skip $\|$ skip with $B \stackrel{l^{\prime}}{\longrightarrow}$ skip. Therefore, also the transition $A^{\prime} \| B \stackrel{l^{\prime \prime}}{\longrightarrow}$ (skip \| skip) \| skip is in the LTS. This is wrong, since $A^{\prime} \| B$ reacts to $\{a\}$ by broadcasting no signal.

We have said in the introduction of this paper that synchronous concurrency cannot be reduced to sequentiality. If this sentence were wrong, it would be possible to simulate agent $A$ of Example 4 by a sequential agent $\hat{A}$. Now, $\hat{A}$ should broadcast both $b$ and $d$ iff both $a$ and $c$ are present. So, both tell $b$ and tell $d$ should appear in the body of both if $a$ then _ and if $c$ then .. In this case, the Gentzen context [ - $\| B$ ], with $B$ as in Example 4, discriminates $A$ and $\hat{A}$, thus proving that $\hat{A}$ cannot simulate $A$.

\section{The Axiomatization}

In this section we present an axiomatization over Gentzen which is sound and complete modulo bisimulation. 


\subsection{The Axiom System}

Let us denote by "=" the least reflexive, symmetric and transitive congruence over Gentzen agents satisfying the axioms in Table 2

Table 2. An axiom system for Gentzen.

$$
\begin{aligned}
& A_{1}\left\|A_{2}=A_{2}\right\| A_{1}\left(\|_{1}\right) \quad A_{0}\left\|\left(A_{1} \| A_{2}\right)=\left(A_{0} \| A_{1}\right)\right\| A_{2} \quad\left(\|_{2}\right) \\
& A\left\|\operatorname{skip}=A \quad\left(\|_{3}\right) \quad A\right\| A=A \quad\left(\|_{4}\right) \\
& \text { if } \gamma \text { then }\left(A_{1} \| A_{2}\right)=\text { if } \gamma \text { then } A_{1} \| \text { if } \gamma \text { then } A_{2} \quad \text { (if_1) } \\
& \text { if } \gamma \text { then if } \gamma^{\prime} \text { then } A=\text { if } \gamma^{\prime} \text { then if } \gamma \text { then } A \\
& \text { if } \gamma \text { then if } \gamma \text { then } A=\text { if } \gamma \text { then } A \\
& A=A \| \text { if } \gamma \text { then } A \\
& \text { if } \gamma \text { then skip }=\text { skip } \\
& \text { next } A=\text { if } a \text { then next } A \| \text { if } \bar{a} \text { then next } A \\
& \text { next } A_{1} \| \operatorname{next} A_{2}=\operatorname{next}\left(A_{1} \| A_{2}\right) \quad \text { (next) } \\
& \operatorname{rec} P . A=A[\operatorname{rec} P . A / P] \quad\left(r e c_{-} 1\right) \\
& B=A[B / P] \text { and } P \text { guarded in } A \text { imply } B=\operatorname{rec} P . A \quad\left(r e c_{-} 2\right)
\end{aligned}
$$

Axioms $\left\|_{1}-\right\|_{3}$, if_1 - if_5, next and rec_1, rec_2 are very intuitive.

Axiom $\|_{4}$ deserves attention. Given transitions $A \stackrel{l_{1}}{\longrightarrow} A_{1}$ and $A \stackrel{l_{2}}{\longrightarrow} A_{2}$ with $l_{1} \neq l_{2}$, it holds that $l_{1} \otimes l_{2} \notin \mathcal{L}$ (this fact can be proved by induction over $A$ ). Moreover, given a label $l \in \mathcal{L}$, it holds that $l \otimes l=l$. From these facts it follows that $A \| A \approx A$, i.e. axiom $\|_{4}$ is sound.

We have explained in Section 2 that one expects axiom if_6, and that one does not expect the following axiom:

$$
\text { tell } b=\text { if } a \text { then tell } b \| \text { if } \bar{a} \text { then tell } b \text {. }
$$

The following result follows from definitions of $\gamma(l), N(\gamma, l), l_{1} \otimes l_{2}$ and $\operatorname{del}(l)$.

Lemma 1 (Soundness). Given agents $A$ and $A^{\prime}, A=A^{\prime}$ implies $A \approx A^{\prime}$.

Our axiom system is finite; all axioms except the recursion specification principle [5 17] (i.e. axiom rec_2) are unconditional. We have not enriched the language by any auxiliary operator. In particular, it is worth noting that we do not need the auxiliary operator "left merge" [6], which, on the contrary, is needed to have finite axiomatizations for asynchronous process algebras (see [18]). 


\subsection{Normal Forms}

Given a string $\vartheta \in(\Pi \cup \bar{\Pi})^{*}$, we denote by if $\vartheta$ then $A$ the agent:

if $\vartheta$ then $A \equiv \begin{cases}A & \text { if } \vartheta=\epsilon(\epsilon \text { denotes the empty string }) \\ \text { if } \gamma \text { then if } \phi \text { then } A & \text { if } \vartheta=\gamma \phi, \phi \in(\Pi \cup \bar{\Pi})^{*}\end{cases}$

Given a string $\vartheta \in(\Pi \cup \bar{\Pi})^{*}$, we denote by $|\vartheta|$ the set $\{\gamma \mid \gamma$ appears in $\vartheta\}$.

Definition 3. An agent $A$ is a normal form if either $A \equiv$ skip or there exist agents $A_{1}, \ldots, A_{n}$ such that:

1. $A \equiv A_{1}\|\ldots\| A_{n}$;

2. $A_{i} \equiv$ if $\vartheta_{i}$ then $B_{i}$, and either $B_{i} \equiv \operatorname{tell} a_{i}$, or $B_{i} \equiv \operatorname{next} A_{i}^{\prime}$, or $B_{i} \equiv P_{i}$, with $a_{i}$ a signal, $A_{i}^{\prime}$ an arbitrary agent and $P_{i}$ a recursion variable;

3. if $B_{i} \equiv$ next $A_{i}^{\prime}$ then, for each $a \in \Pi$, either $a$ or $\bar{a}$ appears in $\vartheta_{i}$;

4. if $B_{i} \equiv$ next $A_{i}^{\prime}$ then every symbol $\gamma \in \Pi \cup \bar{\Pi}$ appears at most once in $\vartheta_{i}$;

5. if $B_{i} \equiv$ next $A_{i}^{\prime}$ and $B_{j} \equiv$ next $A_{j}^{\prime}$ then there exists some $\gamma \in \Pi \cup \bar{\Pi}$ such that $\gamma$ appears in $\vartheta_{i}$ and $\bar{\gamma}$ appears in $\vartheta_{j}$;

6. if $B_{i} \equiv \operatorname{tell} a$ then there exists no $j$ such that $B_{j} \equiv \operatorname{tell}$ a and $\left|\vartheta_{j}\right| \subseteq\left|\vartheta_{i}\right|$.

Let $A \equiv A_{1}\|\ldots\| A_{n}$ be a normal form. Conditions 1, 2 and 3 of Def. 3 will be exploited in proving the completeness of our axiomatization.

Condition 4 is a reasonable request and can be easily respected by transforming agents by axioms if $\_2$ and if_3.

Condition 5 implies that if $A_{i} \equiv$ if $\vartheta_{i}$ then next $A_{i}^{\prime}$ and each signal $a$ such that $a \in\left|\vartheta_{i}\right|$ (resp. $\bar{a} \in\left|\vartheta_{i}\right|$ ) is present (resp. absent), then $A$ will behave as $A_{i}^{\prime}$ at the next instant. More precisely, at the next instant the agent skip $\|$. . || skip $\|$ $A_{i}^{\prime} \|$ skip $\|\ldots\|$ skip will run.

Condition 6 implies that redundant agents broadcasting signals do not appear as parallel components of $A$. Moreover, if $A_{i} \equiv$ if $\vartheta_{i}$ then tell $a$, then $\left(\left|\vartheta_{i}\right|, a\right)$ appears in any label $l$ such that $A \stackrel{l}{\longrightarrow}$, since it is not removed by function del.

We prove now that each agent can be transformed into a normal form, having only normal forms as derivatives, by applying axioms of Table 2 .

Lemma 2 (Reducibility to normal forms). Given an agent $A$, there exist normal forms $A_{1}, \ldots, A_{m}$ such that:

$-A=A_{1}$

- either $A_{i}=$ skip, or $A_{i}=A_{i, 1}\|\ldots\| A_{i, n_{i}}, A_{i, j} \equiv$ if $\vartheta_{i, j}$ then $B_{i, j}$, and, if $B_{i, j} \equiv$ next $A_{f(i, j)}$, then $f_{(i, j)} \in\{1, \ldots, m\}$.

Proof. By structural induction on $A$.

Base case: if either $A \equiv \operatorname{skip}$ or $A \equiv \operatorname{tell} a$ or $A \equiv P$ then the thesis is immediate, because $A$ is a normal form.

Induction step: Assume that, given agents $A^{\prime}$ and $A^{\prime \prime}$, there exist normal forms $A_{1^{\prime}}, \ldots, A_{m^{\prime}}$ and $A_{1^{\prime \prime}}, \ldots, A_{m^{\prime \prime}}$, either $A_{i^{\prime}}=$ skip or $A_{i^{\prime}}=A_{i^{\prime}, 1}\|\ldots\| A_{i^{\prime}, n_{i^{\prime}}}$, either $A_{i^{\prime \prime}}=$ skip or $A_{i^{\prime \prime}}=A_{i^{\prime \prime}, 1}\|\ldots\| A_{i^{\prime \prime}, n_{i^{\prime \prime}}}, A_{i^{\prime}, j} \equiv$ if $\vartheta_{i^{\prime}, j}$ then $B_{i^{\prime}, j}$, $A_{i^{\prime \prime}, j} \equiv$ if $\vartheta_{i^{\prime \prime}, j}$ then $B_{i^{\prime \prime}, j}, A^{\prime}=A_{1^{\prime}}, A^{\prime \prime}=A_{1^{\prime \prime}}$.

We consider the following cases: 
- $A \equiv$ next $A^{\prime}$ : Since $A^{\prime}=A_{1^{\prime}}$ and $=$ is a congruence, we can rewrite $A$ into next $A_{1^{\prime}}$, which does not satisfy Condition 3 of Def. 3 Now, next $A_{1^{\prime}}$ is rewritten into a normal form by axiom if_6, and the thesis follows.

$-A \equiv A^{\prime} \| A^{\prime \prime}$ : Since $A^{\prime}=A_{1^{\prime}}$ and $A^{\prime \prime}=A_{1^{\prime \prime}}$, we infer $A=A_{1^{\prime}} \| A_{1^{\prime \prime}}$ by the fact that $=$ is a congruence. Let us denote with $\mathcal{I} \subseteq\left\{1^{\prime}, \ldots, m^{\prime}\right\} \times$ $\left\{1^{\prime \prime}, \ldots, m^{\prime \prime}\right\}$ the least set such that:

- $\left(1^{\prime}, 1^{\prime \prime}\right) \in \mathcal{I}$;

- if $\left(i^{\prime}, i^{\prime \prime}\right) \in \mathcal{I}, A_{i^{\prime}, j^{\prime}} \equiv$ if $\vartheta_{i^{\prime}, j^{\prime}}$ then next $A_{f\left(i^{\prime}, j^{\prime}\right)}$, $A_{i^{\prime \prime}, j^{\prime \prime}} \equiv$ if $\vartheta_{i^{\prime \prime}, j^{\prime \prime}}$ then next $A_{f\left(i^{\prime \prime}, j^{\prime \prime}\right)}$ and $\left|\vartheta_{i^{\prime}, j^{\prime}}\right|=\left|\vartheta_{i^{\prime \prime}, j^{\prime \prime}}\right|$

then $\left(f\left(i^{\prime}, j^{\prime}\right), f\left(i^{\prime \prime}, j^{\prime \prime}\right)\right) \in \mathcal{I}$.

The set $\mathcal{I}$ contains $\left(i^{\prime}, i^{\prime \prime}\right)$ if $A$ reaches a state in which it behaves as $A_{i^{\prime}} \| A_{i^{\prime \prime}}$. The thesis follows if we infer $A_{i^{\prime}} \| A_{i^{\prime \prime}}=A_{i^{\prime}, i^{\prime \prime}}$, with $A_{i^{\prime}, i^{\prime \prime}}$ an arbitrary normal form having only normal forms as derivatives, for every $\left(i^{\prime}, i^{\prime \prime}\right) \in \mathcal{I}$.

If $A_{i^{\prime}}=\operatorname{skip}\left(\right.$ resp. $A_{i^{\prime \prime}}=$ skip) then we delete it by axiom $\|_{3}$, and the thesis follows. Otherwise, agent $A_{i^{\prime}} \| A_{i^{\prime \prime}}$ satisfies Cond. 1-4 of Def. 3, Let us assume that it does not satisfy Cond. 5, i.e. $A_{i^{\prime}, j^{\prime}} \equiv$ if $\vartheta_{i^{\prime}, j^{\prime}}$ then next $A_{f\left(i^{\prime}, j^{\prime}\right)}$, $A_{i^{\prime \prime}, j^{\prime \prime}} \equiv$ if $\vartheta_{i^{\prime \prime}, j^{\prime \prime}}$ then next $A_{f\left(i^{\prime \prime}, j^{\prime \prime}\right)}$ and $\left|\vartheta_{i^{\prime}, j^{\prime}}\right|=\left|\vartheta_{i^{\prime \prime}, j^{\prime \prime}}\right|$, for some $1 \leq j^{\prime} \leq n_{i^{\prime}}, 1 \leq j^{\prime \prime} \leq n_{i^{\prime \prime}}$. By axioms $\left\|_{1},\right\|_{2}$ we rewrite $A_{i^{\prime}} \| A_{i^{\prime \prime}}$ so that $A_{i^{\prime}, j^{\prime}}$ and $A_{i^{\prime \prime}, j^{\prime \prime}}$ appear as adjacent. Now, by axiom if $\_2$ we rewrite if $\vartheta_{i^{\prime \prime}, j^{\prime \prime}}$ then next $A_{f\left(i^{\prime \prime}, j^{\prime \prime}\right)}$ into if $\vartheta_{i^{\prime}, j^{\prime}}$ then next $A_{f\left(i^{\prime \prime}, j^{\prime \prime}\right)}$, and, by axioms if 1 and next, we rewrite $A_{i^{\prime}, j^{\prime}} \|$ if $\vartheta_{i^{\prime}, j^{\prime}}$ then next $A_{f\left(i^{\prime \prime}, j^{\prime \prime}\right)}$ into if $\vartheta_{i^{\prime}, j^{\prime}}$ then next $\left(A_{f\left(i^{\prime}, j^{\prime}\right)} \| A_{f\left(i^{\prime \prime}, j^{\prime \prime}\right)}\right)$. Since this rewriting preserves conditions 1-4 of Def. 3 and can be applied to every pair $\left(A_{i^{\prime}, j^{\prime}}, A_{i^{\prime \prime}, j^{\prime \prime}}\right)$ such that $\left|\vartheta_{i^{\prime}, j^{\prime}}\right|=\left|\vartheta_{i^{\prime \prime}, j^{\prime \prime}}\right|$, we can transform $A_{i^{\prime}} \| A_{i^{\prime \prime}}$ into an agent satisfying Cond. 1-5 of Def. 3. If the agent so obtained violates Cond. 6 of Def. 3, we can remedy to this situation by applying axioms $\left\|_{1},\right\|_{2}$ as before, and then axioms if _2 and if_4.

- $A \equiv$ if $\gamma$ then $A^{\prime}$ : Since $A^{\prime}=A_{1^{\prime}}$, we infer $A=$ if $\gamma$ then $A_{1^{\prime}}$ by the fact that $=$ is a congruence. If $A_{1^{\prime}}=$ skip then we rewrite if $\gamma$ then $A_{1^{\prime}}$ into skip by axiom if 5 and the thesis follows. Otherwise, since $A_{1^{\prime}}$ is a normal form, we apply axiom if_1 to if $\gamma$ then $A_{1^{\prime}}$ and we obtain an agent satisfying conditions $1,2,3$ and 5 of Def. 3. By axioms if $\_2$ and if $\_3$ we can rewrite every parallel component of this agent so that it satisfies also Cond. 4, while preserving other conditions. The agent so obtained can be transformed into an agent satisfying Cond. 6 of Def. 3 as in the previous case, and the thesis follows.

- $A \equiv \operatorname{rec} P$. $A^{\prime}$ : If $A^{\prime} \equiv$ skip then $A=$ skip by axiom rec_1 and the thesis follows. Otherwise, let $H_{1^{\prime}}, \ldots, H_{m^{\prime}}$ be agents s.t. $H_{i^{\prime}} \equiv A_{i^{\prime}}[A / P]$.

We have $H_{i^{\prime}} \equiv A_{i^{\prime}}[A / P] \equiv\left(A_{i^{\prime}, 1}\|\ldots\| A_{i^{\prime}, n_{i^{\prime}}}\right)[A / P] \equiv$ $A_{i^{\prime}, 1}[A / P]\|\ldots\| A_{i^{\prime}, n_{i^{\prime}}}[A / P] \equiv$

$A_{i^{\prime}, 1}\left[H_{f\left(i^{\prime}, 1\right)} / A_{f\left(i^{\prime}, 1\right)}\right][A / P]\|\ldots\| A_{i^{\prime}, n_{i^{\prime}}}\left[H_{f\left(i^{\prime}, n_{i^{\prime}}\right)} / A_{f\left(i^{\prime}, n_{i^{\prime}}\right)}\right][A / P]=$ $A_{i^{\prime}, 1}\left[H_{f\left(i^{\prime}, 1\right)} / A_{f\left(i^{\prime}, 1\right)}\right]\left[A^{\prime}[A / P] / P\right] \| \ldots$ $\ldots \| A_{i^{\prime}, n_{i^{\prime}}}\left[H_{f\left(i^{\prime}, n_{i^{\prime}}\right)} / A_{f\left(i^{\prime}, n_{i^{\prime}}\right)}\right]\left[A^{\prime}[A / P] / P\right]=$ $A_{i^{\prime}, 1}\left[H_{f\left(i^{\prime}, 1\right)} / A_{f\left(i^{\prime}, 1\right)}\right]\left[A_{1^{\prime}}[A / P] / P\right] \| \ldots$ $\ldots \| A_{i^{\prime}, n_{i^{\prime}}}\left[H_{f\left(i^{\prime}, n_{i^{\prime}}\right)} / A_{f\left(i^{\prime}, n_{i^{\prime}}\right)}\right]\left[A_{1^{\prime}}[A / P] / P\right] \equiv$ $A_{i^{\prime}, 1}\left[H_{f\left(i^{\prime}, 1\right)} / A_{f\left(i^{\prime}, 1\right)}\right]\left[H_{1^{\prime}} / P\right]\|\ldots\| A_{i^{\prime}, n_{i^{\prime}}}\left[H_{f\left(i^{\prime}, n_{i^{\prime}}\right)} / A_{f\left(i^{\prime}, n_{i^{\prime}}\right)}\right]\left[H_{1^{\prime}} / P\right]$, 
where the equalities are inferred by axiom $r e c \_1$ and by the fact that $=$ is a congruence. Now, $H_{i^{\prime}}$ has been rewritten as a parallel composition of normal forms having only normal forms as derivatives. Let us call $K_{i^{\prime}}$ such agent. Every $K_{i^{\prime}}$ can be transformed in a normal form as in the case for "||". Finally, the thesis follows since $A=A^{\prime}[A / P]=A_{1^{\prime}}[A / P] \equiv H_{1^{\prime}}=K_{1^{\prime}}$.

\subsection{The Proof of Completeness}

First of all we recall the notion of guarded recursive specification and we prove that every guarded recursive specification has a solution which is unique modulo $=$. Then, we exploit this result and Lemma 2 to prove that two arbitrary agents $A$ and $A^{\prime}$ such that $A \approx A^{\prime}$ are equated by our axioms. In fact, we prove that $A=B$ and $A^{\prime}=B^{\prime}$, where $A^{\prime}$ and $B^{\prime}$ are normal forms and solutions of the same guarded recursive specification.

A recursive specification over variables $\vec{P}=P_{1}, \ldots, P_{m}$ is a set of equations

$$
P_{i}=B_{i} \quad 1 \leq i \leq m
$$

where $B_{i}$ is an agent, $1 \leq i \leq m$. It is guarded if and only if $P_{1}, \ldots, P_{m}$ are guarded in $B_{1}, \ldots, B_{m}$, i.e. $P_{1}, \ldots, P_{m}$ appear in $B_{1}, \ldots, B_{m}$ in bodies of next _.

A solution of a recursive specification as (1) is a set of agents $\vec{A} \equiv A_{1}, \ldots, A_{m}$ such that $A_{i}=B_{i}[\vec{A} / \vec{P}], 1 \leq i \leq m$.

The following result, which states that every guarded recursive specification has a solution unique modulo "=", has been presented in [17]. (Note that axiom $r e c \_2$ plays a central rôle in the proof.)

Lemma 3. Every guarded recursive specification

$$
P_{i}=B_{i} \quad 1 \leq i \leq m
$$

has a solution $\vec{A} \equiv A_{1}, \ldots, A_{m}$. Given any other solution $\overrightarrow{A^{\prime}} \equiv A_{1}^{\prime}, \ldots, A_{m}^{\prime}$, it holds that $A_{i}^{\prime}=A_{i}, 1 \leq i \leq m$.

We show now that two arbitrary bisimilar agents are equated by our axioms.

Lemma 4 (Completeness). Given agents $A$ and $A^{\prime}, A \approx A^{\prime}$ implies $A=A^{\prime}$.

Proof. By Lemma 2, there are normal forms $A_{1}, \ldots, A_{m}$ and $A_{1^{\prime}}, \ldots, A_{m^{\prime}}$ s.t.:

- $A=A_{1}, A^{\prime}=A_{1^{\prime}}$

- either $A_{i}=$ skip or $A_{i}=A_{i, 1}\|\ldots\| A_{i, n_{i}}, 1 \leq i \leq m$

- either $A_{i^{\prime}}=\operatorname{skip}$ or $A_{i^{\prime}}=A_{i^{\prime}, 1}\|\ldots\| A_{i^{\prime}, n_{i^{\prime}}}, 1 \leq i^{\prime} \leq m^{\prime}$

- $A_{i, j} \equiv$ if $\vartheta_{i, j}$ then $B_{i, j}, 1 \leq i \leq m, 1 \leq j \leq n_{i}$

- $A_{i^{\prime}, j^{\prime}} \equiv$ if $\vartheta_{i^{\prime}, j^{\prime}}$ then $B_{i^{\prime}, j^{\prime}}, 1 \leq i^{\prime} \leq m^{\prime}, 1 \leq j^{\prime} \leq n_{i^{\prime}}$. 
Moreover, by axioms $\|_{4}$ we can assume that $A_{i, j} \not \equiv A_{i, h}$ for $1 \leq j<h \leq n_{i}$ and $A_{i^{\prime}, j^{\prime}} \not \equiv A_{i^{\prime}, h^{\prime}}$ for $1 \leq j^{\prime}<h^{\prime} \leq n_{i^{\prime}}$.

Now, to prove the thesis it is sufficient to prove that $A_{1}=A_{1^{\prime}}$.

Let us denote by $\mathcal{I} \subseteq\{1, \ldots, m\} \times\left\{1^{\prime}, \ldots, m^{\prime}\right\}$ the set of pairs $\left(i, i^{\prime}\right)$ such that $A_{i} \approx A_{i^{\prime}}$. Since $A \approx A^{\prime}, A=A_{1}$ and $A^{\prime}=A_{1^{\prime}}$, and $=$ is sound modulo $\approx$, we have $\left(1,1^{\prime}\right) \in \mathcal{I}$.

We prove now that, given a pair $\left(i, i^{\prime}\right) \in \mathcal{I}$, and an arbitrary index $1 \leq j \leq n_{i}$, there exists an index $1 \leq j^{\prime} \leq n_{i^{\prime}}$ such that:

$-\vartheta_{i^{\prime}, j^{\prime}}=\vartheta_{i, j}$

- either $B_{i, j} \equiv B_{i^{\prime}, j^{\prime}}$, or $B_{i, j} \equiv$ next $A_{f(i, j)}, B_{i^{\prime}, j^{\prime}} \equiv$ next $A_{f\left(i^{\prime}, j^{\prime}\right)}$ and $\left(f(i, j), f\left(i^{\prime}, j^{\prime}\right)\right) \in \mathcal{I}$.

We begin with proving that there is a $1 \leq j^{\prime} \leq n_{i^{\prime}}$ such that $\left|\vartheta_{i, j}\right|=\left|\vartheta_{i^{\prime}, j^{\prime}}\right|$ and the second condition above is satisfied. We consider the following cases:

- $B_{i, j} \equiv$ tell $a$ : Since $A_{i}$ is a normal form and, in particular, it satisfies Cond. 6 of Def. 3, $\left(\left|\vartheta_{i, j}\right|, a\right)$ appears in any label $l$ such that $A \stackrel{l}{\longrightarrow}$. Since $A_{i} \approx A_{i^{\prime}}$, we have $A_{i^{\prime}} \stackrel{l}{\longrightarrow}$. Since $\left(\left|\vartheta_{i, j}\right|, a\right)$ appears in $l$, there exists $1 \leq j^{\prime} \leq n_{i^{\prime}}$ such that $\left|\vartheta_{i^{\prime}, j^{\prime}}\right|=\left|\vartheta_{i, j}\right|$ and $B_{i^{\prime}, j^{\prime}} \equiv$ tell $a$.

- $B_{i, j} \equiv$ next $A_{f(i, j)}$ : Since $A_{i}$ is a normal form and, in particular, it satisfies Cond. 5 of Def. 3 , we have $A \stackrel{l}{\longrightarrow} A_{f(i, j)}$ for some label $l$. Since $A \approx A^{\prime}$, there exists an agent $A_{f\left(i^{\prime}, j^{\prime}\right)}$ such that $A \stackrel{l}{\longrightarrow} A_{f\left(i^{\prime}, j^{\prime}\right)}$ and $A_{f(i, j)} \approx A_{f\left(i^{\prime}, j^{\prime}\right)}$.

Now, if $\left|\vartheta_{i, j}\right|=\left|\vartheta_{i^{\prime}, j^{\prime}}\right|$ but $\vartheta_{i, j} \neq \vartheta_{i^{\prime}, j^{\prime}}$, we can apply axiom if_2 to $A_{i^{\prime}, j^{\prime}}$ while preserving conditions of Def. 3. So, we can rewrite if $\vartheta_{i^{\prime}, j^{\prime}}$ then $B_{i^{\prime}, j^{\prime}}$ into if $\vartheta_{i, j}$ then $B_{i^{\prime}, j^{\prime}}$. Now, we reapply our reasoning from the beginning.

Let us consider now the recursive specification

$$
P_{i, i^{\prime}}=A_{i, i^{\prime}} \quad\left(i, i^{\prime}\right) \in \mathcal{I}
$$

where $A_{i, i^{\prime}}$ is the agent such that $A_{i, i^{\prime}} \equiv H_{i, i^{\prime}, 1}\|\ldots\| H_{i, i^{\prime}, n_{i}}$, with either $H_{i, i^{\prime}, j} \equiv$ if $\vartheta_{i, j}$ then next $P_{f(i, j), f\left(i^{\prime}, j^{\prime}\right)}$, if both $A_{i, j} \equiv$ if $\vartheta_{i, j}$ then next $A_{f(i, j)}$ and $A_{i^{\prime}, j^{\prime}} \equiv$ if $\vartheta_{i, j}$ then next $A_{f\left(i^{\prime}, j^{\prime}\right)}$, or $H_{i, i^{\prime}, j} \equiv A_{i, j} \equiv A_{i^{\prime}, j^{\prime}}$, otherwise.

This recursive specification is guarded and has both $\vec{G}$ and $\overrightarrow{G^{\prime}}$ as solutions, where $G_{i, i^{\prime}} \equiv A_{i}$ and $G_{i, i^{\prime}}^{\prime} \equiv A_{i^{\prime}}$ for each $\left(i, i^{\prime}\right) \in \mathcal{I}$. Now, by Lemma 3 it follows that $A_{i}=A_{i^{\prime}},\left(i, i^{\prime}\right) \in \mathcal{I}$. Since $\left(1,1^{\prime}\right) \in \mathcal{I}$, we have $A_{1}=A_{1^{\prime}}$, as required.

The following theorem states that axioms in Table 2 give an axiomatization sound and complete modulo bisimulation on Gentzen agents.

Theorem 2 (Soundness and completeness). Given arbitrary agents $A$ and $A^{\prime}, A=A^{\prime}$ if and only if $A \approx A^{\prime}$.

Proof "If": by Lemma 4, "Only if": by Lemma 1 . 


\section{Conclusions and Future Work}

We have presented an axiomatization of the synchronous language Gentzen, in order to characterize behaviorally equivalent agents.

The axiomatization of Gentzen cannot be done by trivially adapting techniques developed in the field of asynchronous concurrency. Axiomatizations of asynchronous process description languages are based on the transformation of processes into "head normal forms". In order to transform concurrent processes into head normal forms, concurrency must be simulated by interleaving. This is impossible in the synchronous setting, because processes running in parallel are perfectly synchronized and cannot interleave.

The axiomatization of Gentzen should be intended as an initial step toward the development of axiomatizations for state-oriented synchronous languages. In fact, Gentzen offers all basic constructs that are shared by the languages in this class and that distinguish synchronous languages w.r.t. asynchronous ones.

Given any other state-oriented synchronous language, we believe that it can be axiomatized by simply extending our work to deal with those constructs that are typical in such a language and that are not offered by Gentzen. In particular, we believe that such a language can be axiomatized by exploiting the notion of normal form proposed in this paper. To support this affirmation, we have developed an axiomatic semantics for the language Esterel [8] in [26]. The axiomatization of Esterel is derived by extending the technique proposed in the present paper to deal with the interruption mechanism "trap", the suspension mechanism "suspend", the construct of instantaneous sequencing ";" and the property of constructiveness [7]. The technique adopted to axiomatize these constructs is to express them in terms of constructs of Gentzen.

The approach of 26] can be generalized to any synchronous language: given a superset of Gentzen, it is sufficient to express any construct in terms of constructs of Gentzen. So, Gentzen could play in the synchronous setting the rôle of $T_{\text {fintree }}$ (i.e. a subset of CCS suitable for expressing finite LTSs) in the asynchronous setting, where an axiomatization of $T_{\text {fintree }}$ has been proposed in [17], and supersets of $T_{\text {fintree }}$ are axiomatized by expressing any construct in terms of constructs of $T_{\text {fintree }}$. Following [1,2, where algorithmic techniques to compute axiomatizations of classes of supersets of $T_{\text {fintree }}$ have been proposed, one could develop algorithmic techniques to compute axiomatizations of classes of supersets of Gentzen.

\section{References}

1. Aceto, L.: Deriving complete inference systems for a class of GSOS languages generating regular behaviors. Proc. of CONCUR '94, Springer LNCS 836, 1994.

2. Aceto, L., Bloom, B. and Vaandrager, F.: Turning SOS rules into equations. Information and Computation 111, 1994.

3. André, C.: Representation and analysis of reactive behaviors: a synchronous approach. Presented at CESA '96, IEEE-SMC, Lille, France, 1996. 
4. Benveniste, A. and Berry, G. (editors): Another look at real-time systems. Proceedings of the IEEE 79, 1991.

5. Bergstra, J.A. and Klop, J.W.: A complete inference system for regular processes with silent moves. Proc. of Logic Colloquium 1986, North Holland, 1988.

6. Bergstra, J.A. and Klop, J.W.: Process algebra for synchronous communication. Information and Computation 60, 1984.

7. Berry, G.: The constructive semantics of pure Esterel. Version 3.0, 1999. URL: http://www.inria.fr/meije/personnel/Gerard.Berry.html.

8. Berry, G. and Gonthier, G.: The Esterel synchronous programming language: design, semantics, implementation. Science of Computer Programming 19, 1992.

9. Caspi, P., Halbwachs, N., Pilaud, P. and Plaice, J.: Lustre, a declarative language for programming synchronous systems. Proc. of POPL '87, ACM Press, 1987.

10. Gonthier, G: Sémantique et modèles d'exécution des langages réactifs synchrones; application à Esterel. Thèse d' informatique, Univesité d'Orsay, France, 1988.

11. Halbwachs, N.: Synchronous programming of reactive systems. Kluwer Academic Publishers, Dordrecht, 1993.

12. Harel, D.: Statecharts: A visual formalism for complex systems. Science of Computer Programming 8, 1987.

13. Harel, D. and Naamad, A.: The Statemate semantics of Statecharts. ACM Transactions on Software Engineering Methodologies 5, 1996.

14. Harel, D. and Pnueli, A.: On the development of reactive systems. In K.R. Apt editor, Logic and Models of Concurrent Systems, NATO, ASI-13, Springer, 1985.

15. Huizing, C. and Gerth, R.: Semantics of reactive systems in abstract time. Proc. of REX Workshop "Real Time: Theory in Practice", Springer LNCS 600, 1992.

16. Maraninchi, F.: Operational and compositional semantics of synchronous automaton composition. Proc. of CONCUR '92, Springer LNCS 630, 1992.

17. Milner, R.: A complete inference system for a class of regular behaviors. Journal of Computer and System Sciences 28, 1984.

18. Moller, F.: The importance of the left merge operator in process algebras. Proc. of ICALP '90, Springer LNCS 443, 1990.

19. Plotkin, G.: A structural approach to operational semantics. Technical Report DAIMI FN-19, University of Aarhus, Denmark, 1981.

20. Pnueli, A. and Shalev, M.: What is a step: On the semantics for Statecharts. Proc. of TACS '91, Springer LNCS 526, 1991.

21. Saraswat, V.A., Jagadeesan, R. and Gupta, V.: Default timed concurrent constraint programming. Proc. of POPL '95, ACM Press, 1995.

22. Saraswat, V.A., Jagadeesan, R. and Gupta, V.: Timed default concurrent constraint programming. Journal of Symbolic Computation 11, 1996.

23. Scholtz, P., Nazareth, D. and Regensburger, F.: Mini-Statecharts: A compositional way to model parallel systems. In $9^{\text {th }}$ Int. Conf. on Parallel and Distributed Computing Systems, Dijon, France, 1997.

24. de Simone, R.: Higher level synchronizing devices in SCCS-Meije. Theoretical Computer Science 37, 1985.

25. Tini, S.: On the expressiveness of Timed Concurrent Constraint Programming. Proc. of Express '99, Electronic Notes in Theoretical Computer Science 27, 1999.

26. Tini, S.: Structural Operational Semantics for Synchronous Languages. Ph.D. Thesis, Dipartimento di Informatica, University of Pisa, Italy, 2000. 